\title{
Mise en œuvre et interprétation de la comparaison de modèles
}

\author{
Th. Leviandier
}

\author{
CEMAGREF
}

\section{Objectifs}

La multiplicité des modèles existant en hydrologie induit assez naturellement des idées de comparaison. Nous discuterons des modalités et difficultés de ces tentatives, qui même si elles sont présentées en termes généraux, se réfèrent plus spécialement aux modèles pluie-débit, dont la prolifération est vigoureuse. Les modèles de propagation, sans doute moins différents entre eux dans leurs principes, sont également justiciables d'intercomparaison. Par ailleurs, l'exposé concernera surtout l'utilisation en temps réel des modèles, qui est le problème d'actualité même si tous les problèmes de la simulation sont loin d'être réglés.

L'objectif général d'évaluation au moyen de la comparaison peut être nuancé selon deux points de vue principaux. On peut avoir une vision strictement opérationnelle selon laquelle c'est le résultat final qui compte : on juge à la fois le modèle, la procédure de correction en temps réel, l'aptitude du programme informatique et de l'utilisateur à intégrer des aléas comme des erreurs grossières de données. On peut au contraire chercher à évaluer les performances de l'algorithme dans les meilleures conditions possibles, représentatives de la réalité, mais en simplifiant éventuellement pour faciliter l'analyse.

Dans le premier cas, on pourra tolérer des corrections manuelles quelque peu subjectives, dans le second l'utilisation d'algorithmes exigeant des temps de calcul prohibitifs. La première approche livre l'état actuel des techniques, la seconde éclaire peut-être mieux les progrès futurs, si du moins l'on admet que la mise en œuvre est plus facile à améliorer que les aspects fondamentaux des modèles.
Il serait assez naif de croire que d'une comparaison bien conduite pourrait émerger un modèle qui surclasserait les autres et auquel tous les praticiens et tous les développeurs de modèles se rallieraient. Le caractère de compétition peut être cependant plus ou moins marqué, les utilisateurs étant sans doute plus préoccupés par le choix d'un modèle, les développeurs par l'existence de références qui leur permettent de détecter et de corriger les défaillances de leurs modèles, les écarts des résultats d'un seul modèle par rapport à la réalité pouvant toujours être considérés comme irréductibles et imputés aux données.

\section{Conditions pratiques de la comparaison}

La comparaison peut être effectuée en situation réelle, mais cela semble difficile à organiser à l'heure actuelle, ou en simulation totale de temps réel, c'est-à-dire en fournissant les valeurs réelles correspondant aux prévisions après que ces prévisions aient réellement été émises, ou bien encore en simulation de temps réel sur fichier continu, les données observées étant fournies d'avance mais ne devant évidemment pas être utilisés.

Par ailleurs, on peut demander à chaque utilisateur habituel ou développeur de faire fonctionner son modèle, où le faire faire par un service ou un chercheur unique après avoir rassemblé les programmes informatiques ou reprogrammé l'ensemble.

Il est assez clair que le jugement du caractère opérationnel sera mieux satisfait par une simulation totale de temps réel par des utilisateurs différents sur chaque modèle et que la compréhension du fonctionne-

\section{Implementation and interpretation of comparison concerning models}

The comparison of models is a necessary stage of advanced techniques and which has just been demonstrated on a French scale, for example on the Meurthe, and on a worldwide scale under the sponshorship WMO and which is continuing. The objectives of the attempts, the methological precautions to be taken, the selection of criterium and the problem concerning implementation and interpretation are examined. 
ment des différents modèles sera mieux servie par une mise en œuvre unique sur une longue période donc en simulation de temps réel sur fichier continu. La première démarche est celle de l'Organisation Météorologique Mondiale (OMM) qui a organisé un atelier de travail à Vancouver en août 1987; la seconde est plus usuelle car elle peut être entreprise à l'initiative de tout chercheur et il est généralement préférable de présenter un nouveau modèle comme une alternative (meilleure!) à un modèle existant. Pour avoir une certaine ampleur et concerner un nombre appréciable de modèles ou variantes, ce genre d'opération assez lourde doit toutefois entrer dans un projet bien défini, tel ceux dans la mouvance du groupe S.H.F. de prévisions de crues, ou, et c'est à celle là que nous nous référerons, pour y avoir participé, la comparaison de modèles effectuée par le L.N.H. (E.D.F.) et le CEMAGREF avec la participation financière du Ministère de l'Environnement.

Enfin, il est souhaitable que ces comparaisons s'appuient sur le plus grand nombre possible de bassins versants. En fait la mise en œuvre de modèles reste à l'heure actuelle relativement difficile et les comparaisons en cours portent sur un petit nombre de cas, 3 bassins versants par exemple dont 2 en Amérique du Nord et l'Orgeval (Seine et Marne) géré par le CEMAGREF pour le projet OMM.

\section{Sorties des modèles faisant l'objet de comparaison}

\subsection{Période continue ou sélection d'événements}

Pour garantir la meilleure objectivité et représentativité, il est préférable d'évaluer les modèles sur une longue période continue. Si l'on s'intéresse seulement aux crues, et particulièrement en cas de simulation totale de temps réel, pour de simples considérations matérielles, on est obligé de sélectionner au-dessus d'un seuil, que l'on devrait mais qu'on ne fait pas, prendre par rapport aux pluies.

Il est vrai que le contrôle sur une longue période, que l'on pourrait qualifier de point de vue "statistique ", tend à faire négliger le suivi individuel des crues, qui permet de mieux percevoir les mécanismes de corrections.

Lorsqu'on sélectionne un petit nombre d'événements, on peut vouloir les choisir représentatifs, où au contraire sélectionner des événements à priori difficiles (crues complexes, premières crues après les étiages, crues correspondant à des pluies anormalement réparties spatialement).

C'est ce dernier choix par exemple qui a été fait dans le projet OMM; il est excellent pour la mise à l'épreuve des modèles (comme prestation offerte aux développeurs) mais réduit sans doute la portée des conclusions sur les comparaisons intermodèles car les modèles réagissent sans doute différemment à différents types de difficultés et il faut une grande expérience du bassin pour choisir les cas réellement difficiles sans être totalement abérants et non représentatifs.

\subsection{Prévisions à échéance supérieure au temps de réponse des pluies}

On peut vouloir prolonger le délai de prévision du modèle au-delà du temps de réponse des pluies, en y incorporant des prévisions quantitatives de précipitations. S'il n'y a pas de raison de penser que des modèles différents doivent être utilisés dans cette variante, les procédures de correction en temps réel qui, idéalement, dépendent des incertitudes identifiées, peuvent devoir être adaptées et il est bon de les tester.

Comme on dispose rarement aujourd'hui de ces prévisions quantitatives (distribuées dans le temps et dans l'espace avec la précision nécessaire pour le modèle) on utilise à la place des prévisions parfaites. On tend ainsi à s'écarter des conditions de prévision pour faire de la simulation avec réajustement glissant des conditions initiales. Mon opinion est que ce test n'est représentatif d'aucun problème concret et que faire fonctionner des procédures de correction plus longtemps, mais avec une hypothèse d'erreurs très irréaliste, donne une information difficilement exploitable sur leur comportement (une borne supérieure), surtout en comparaison intermodèles.

Si l'on dispose de prévisions réelles de pluies, il est tout à fait légitime de les inclure dans les entrées. On peut éventuellement tester des simulations de prévisions (prévisions de persistance) mais l'exercice est un peu académique.

\subsection{Prévisions à plusieurs échances}

Les modèles peuvent généralement être utilisés pour donner des prévisions à différents pas de temps. Parfois il s'agira de variantes du modèle avec des entrées différentes. Notons que pour une prévision à court terme on peut avoir intérêt à renoncer au modèle pluie-débit pour utiliser une station de débit amont.

Une complication assez notable est que les procédures de correction en temps réel dépendent de l'échéance et que la trajectoire des prévisions corrigées à échéances croissantes, calculées indépendemment, ne constitue pas un hydrogramme de sortie du modèle. Ce fait peut être, ou non, considéré comme génant, et on peut préférer une prévision d'hydrogramme global, plus crédible, même si chacun de ses points n'est pas optimal.

Avant d'approfondir ce point, rappelons les différents types de corrections en temps réel, dont la justification théorique (et presque philosophique) est loin de faire l'objet d'un consensus :

- correction des entrées du modèle (le dernier recours?);

- correction de l'état du système (assez courante grâce aux théories du filtrage, surtout du filtrage linéaire appliqué de façon rigoureuse à des systèmes linéaires, et souvent de façon approchée mais performante à des systèmes non linéaires);

- correction des sorties du modèle (la plus facile);

- correction des paramètres du modèle (pratiquement limitée aux modèles linéaires).

Notons - les estimations de vecteurs d'état et ^ les prévisions à 1 pas de temps et multiplions ces symboles 
pour les prévisions à $n$ pas de temps et les vecteurs d'état utilisés pour ces prévisions.

L'indice désignant le temps, $F$ l'opérateur modèle et $G$ un terme (à optimiser) mesurant l'intensité des corrections, l'évolution de l'état du système s'écrit :

$$
\begin{aligned}
& \bar{X} 2=F 1 \bar{X}_{1}+G 1\left({ }^{\wedge} Y 1-Y 1\right) \\
& \bar{X} 3=F 2 \bar{X} 2+G 2\left({ }^{\wedge} Y 2-Y 2\right)
\end{aligned}
$$

on peut ne corriger le modèle qu'en fonction de la prévision à 1 pas de temps et le laisser évoluer sans correction pour les prévisions plus lointaines :

$$
\overline{\bar{X}} 3=F 2 \bar{X} 2
$$

(on pourrait optimiser pour une autre échéance différente de 1)

on peut corriger de façon optimale la prévision à 2 pas de temps, sans tenir compte de la correction à un pas de temps :

$$
\overline{\bar{X}} 3=F 2 F 1 \overline{\bar{X}}_{1}+\mathrm{Ga} 1\left({ }^{*} Y 1-Y 1\right)
$$

ou appliquer le modèle à partir de la prévision à un pas de temps et corriger en fonction de la dernière erreur connue sur la prévision à 2 pas de temps :

$$
\overline{\bar{X}} 3=F 2 \bar{X} 2+\mathrm{Gb} 1\left({ }^{\wedge} Y 1-Y 1\right)
$$

ou encore adopter une solution intermédiaire :

$$
\overline{\bar{X}} 3=F 2 F 1 X 1+\operatorname{Gc} 1\left({ }^{\wedge} Y 1-Y 1\right)
$$

On voit que le choix est ouvert. Malheureusement, si ces solutions diffèrent dans la complexité du filtrage, qui n'est pas la partie consommatrice de temps calcul, toutes à l'exception de la première, exigent un grand nombre d'applications du modèle. On peut alors penser corriger l'état de système pour une échéance et les sorties pour les autres.

Il est vraisemblable que la plupart des modèles, n'étant pas normalement utilisés pour une large gamme d'échéances, ne sont pas dotés de procédures très sophistiquées et très coûteuses en calcul pour des corrections optimales (en un sens à définir) à toutes les échéances.

L'évaluation des modèles risque donc d'être influencée par des maladresses qui portent sur des points relativement accessoires.

Il me semble qu'il serait bien plus simple de se contenter d'une comparaison à une échéance voisine du temps de réponse des pluies.

\section{Critères}

Les critères dépendent des applications pratiques; ainsi, un critère sur le volume total d'une crue peut être très important ou tout à fait inutile.

On s'accorde généralement à utiliser comme premier critère numérique un critère d'erreur quadratique, ou coefficient de détermination. Il est aussi apprécié, surtout en prévision, d'avoir un critère analogue, non sur les débits bruts, mais sur les accroissements. De façon générale, un critère significatif sans correction de temps réel ne l'est pas toujours avec correction.

Le corrélogramme des erreurs est également utile pour voir si toute l'information a bien été exploitée.

Il serait utile d'avoir un critère sur les maxima mais il est plus difficile à élaborer.

Bien d'autres critères ont été proposés mais on renonce généralement à les exploiter quand on a beaucoup de modèles. Notons que le problème pourrait être reposé quand on franchira un degré de complexité en utilisant un grand nombre de bassins et qu'un traitement de masse de ces critères sera de toute façon nécessaire. Plutôt que d'élaborer des critères complexes, on peut utiliser des critères simples sur des périodes bien choisies. L'intérêt de distinguer les crues des décrues est souvent mis en avant, mais ce n'est pas très facile à appliquer.

En fait l'essentiel des jugements se fait sur des représentations graphiques. Notons que dans le cas de prévisions à plusieurs échéances, la dimension du problème (modèle, date d'émission de la prévision, date de vérification + donnée dépendante : délai) est source de débat entre les différentes représentations possibles.

\section{Quelques très brefs résultats}

A titre d'illustration, nous donnons deux des meilleurs graphiques de résultats parmi une trentaine de modèles ou variantes essayés pour la prévision de la Meurthe à Dammelevières au pas de temps journalier.

Les deux modèles, de principes tout à fait différents, donnent des résultats de qualité équivalente. Peut-on en tirer une conclusion définitive ? Les données de contrôle ne sont pas très abondantes et il n'est pas facile d'établir de critères de significativité dans ce domaine. Le modèle stochastique est l'un parmi de nombreuses variantes que l'on aurait été bien incapable de choisir à priori (détail de la structure d'erreur, correction de paramètres plutôt que correction de l'état du système). Il est possible que le hasard ait avantagé ce modèle (par de "bonnes" erreurs en début des crues. Le modèle à réservoir est également l'un parmi plusieurs, mais ses retouches successives ont été conçues dans un esprit plus déterministe. Peut-on admettre qu'il faille moins de données pour valider un tel modèle? A performances égales sur un jeu de contrôle, on lui accordera sûrement plus de crédit pour extrapoler dans des conditions exceptionnelles. On sait par ailleurs que ce modèle est utilisable à des pas de temps beaucoup plus courts alors que c'est beaucoup plus difficile pour des modèles régressifs ou dérivés.

D'un autre côté, la méthode de correction des paramètres, sous la réserve exprimée plus haut, semble intéressante et son étude mérite d'être poursuivie, en particulier pour les modèles à réservoirs où elle présente de plus grandes difficultés techniques.

La comparaison débouche donc sur des résultats, peut être encore insuffisamment validés, mais aussi sur des questions aussi importantes pour des progrès futurs. L'étude ne s'est d'ailleurs pas déroulée comme une comparaison de modèles figés, mais en incorporant des améliorations. 
JEU VARIABLES MEURTIE-REGR1
MODELE
MA1 $Q(+2)=.707 \underset{c}{.}=.156 .0736 \mathrm{P}(-0)+.1389 \mathrm{P}(-1)+1.2827$ (initial)
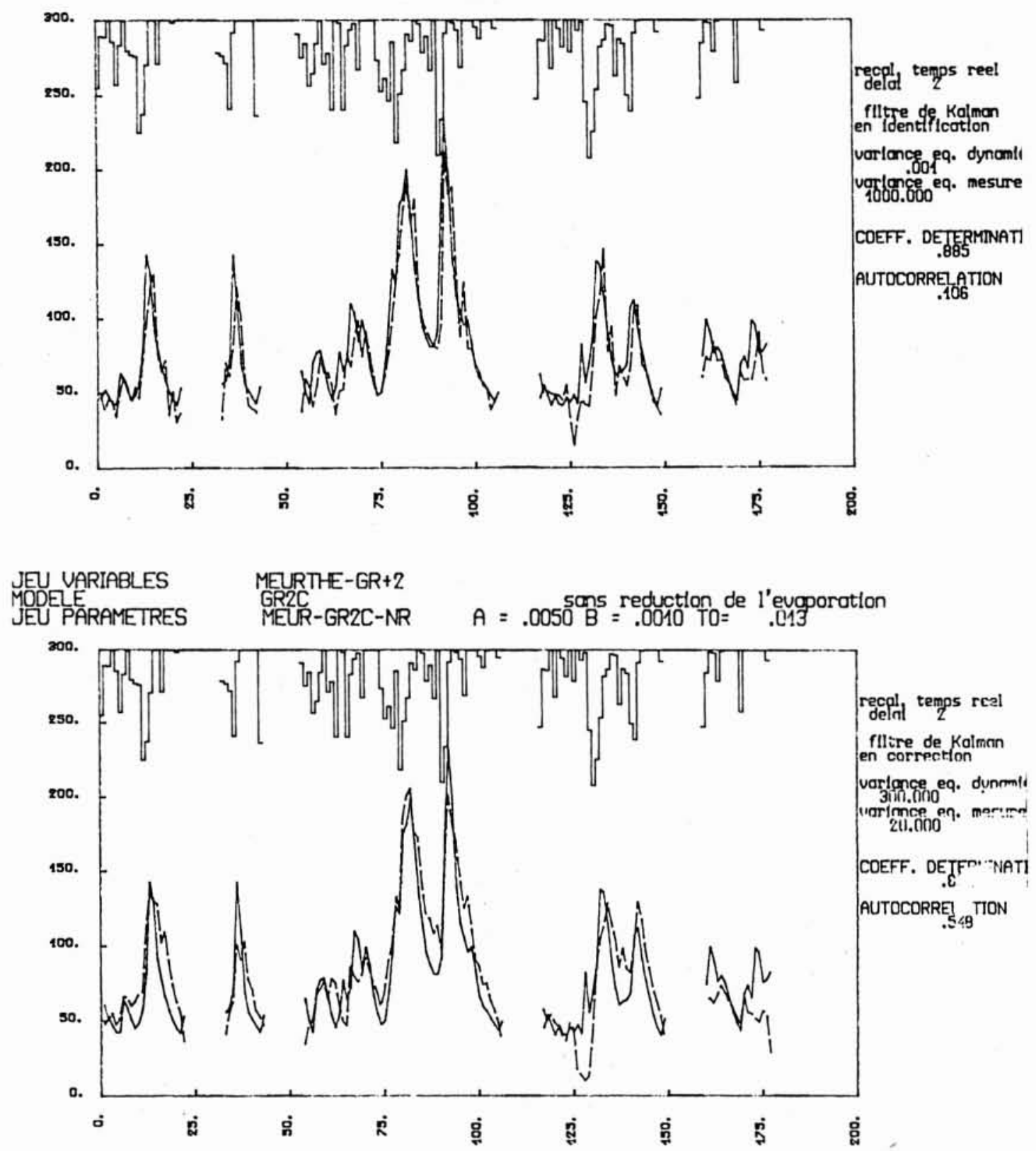

L'ambition était bien au départ d'étudier des modèles opérationnels, mais il y a loin entre opérationnels au niveau de la littérature et réellement disponibles sous forme de programmes informatiques avec une expertise suffisante. Dans le cas d'une comparaison d'outils réellement opérationnels, il faut au contraire avoir un protocole très bien défini et s'en écarter le moins possible.
Adresse de l'auteur

Monsieur Th. Leviandier

CEMAGREF

Parc de Tourvoie

92160 Antony

Tél. : 46.66.21.09 